\title{
A Theoretical and Practical Course on Urine Microscopy in Nigeria: A Model for Nephrological Skill Transfer
}

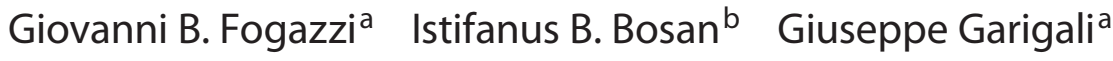 \\ Fatiu Arogundade ${ }^{c}$ \\ ${ }^{a}$ Research Laboratory on Urine, Unità Operativa di Nefrologia Dialisi, Trapianto, Fondazione IRCCS Ca' Granda, \\ Ospedale Maggiore Policlinico, Milano, Italy; ${ }^{b}$ Department of Medicine, Ahmadu Bello University Teaching \\ Hospital, Zaria, and ${ }^{\mathrm{C}}$ Department of Medicine, Obafemi Awolowo University, Ile-Ife, Nigeria
}

\section{Key Words}

Urine microscopy $\cdot$ Urinary sediment $\cdot$ Urinary sediment teaching

\begin{abstract}
Background: In the developing world, the diagnostic power of nephrologists is heavily limited by financial, technical and human resource constraints. Urine microscopy (UM) is a basic, inexpensive and relatively simple diagnostic tool, which supplies irreplaceable information. Recently, a theoretical and practical course on UM was organized during the 22nd annual meeting of the Nigerian Society of Nephrology. Methods: The 2-day course was based on power point presentations and on examination of true urine samples by means of a microscope equipped with phase contrast, polarized light and a video camera for projection of the findings. Results: The presentations described were the methodology, the particles of the urine sediment and their clinical interpretation, the urine sediment in different clinical conditions, and 12 clinical cases, which demonstrated the value of UM in clinical practice. Practical sessions showed the most important urine particles, and how they could be identified and combined into urine profiles. More than $97 \%$ of the partici-
\end{abstract}

pants found the course to be useful and practicable and a UM program was actually started in 1 Nigerian center a few days after the course. Conclusion: This course demonstrated that nephrological skills can be transferred from the developed to the developing world without large financial investments.

Copyright $\odot 2010$ S. Karger AG, Basel

\section{Introduction}

The course was conceived in May 2009 by a representative of the Nigerian Society of Nephrology (F.A.) and G.B.F., a former member of the Subcommittee for Africa of ISN/COMGAN and an expert in the field of urine microscopy [1,2] and its teaching [3].

The course, which was organized as a pre-Congress event of the 22nd Annual Conference of the Nigerian Society of Nephrology, had the support of ISN/COMGAN and took place at Ahmadu Bello University Teaching Hospital in Zaria, Kaduna State, Nigeria, on February 13 and 14, 2010.

The aim of the course was to demonstrate the importance of urine sediment examination and encourage its

Giovanni B. Fogazzi

Unità Operativa di Nefrologia, Fondazione IRCCS Ca’ Granda

Ospedale Maggiore Policlinico, Via Commenda 15

IT-20122 Milano (Italy)

Tel. +39 025503 6331, Fax +39 025503 4550, E-Mail fogazzi@ policlinico.mi.it 
practice by nephrologists. Consequently, the course outline included methodology of urine sediment examination, identification and interpretation of urine sediment particles and urine profiles found in a wide range of diseases.

\section{Methods}

The course was planned to be both theoretical and practical, based on oral presentations and the examination of true urine samples with a microscope and the projections of the findings on a large screen by means of a video camera.

The microscope, a Nikon Eclipse 50i (Nikon, Kanagawa, Japan) equipped with 2 magnifications $(\times 200$ and $\times 400)$, bright field, phase contrast and polarized light devices, was transported for the purpose from Italy. Similarly, urine samples which had been selected and prepared in the laboratory of the renal unit of Ospedale Maggiore Policlinico of Milan were transported [3]. The detailed methodology of preparation of urine sediment is described as follows: 10 -ml aliquots of the second urine of the morning from different patients were centrifuged for $10 \mathrm{~min}$ at 2,000 $\mathrm{rpm}$. Then, after removal of $9.0 \mathrm{ml}$ of the supernatant urine by a pump, the pelleted sediment was gently but thoroughly resuspended with a Pasteur pipette in the $1.0 \mathrm{ml}$ of urine left. Then, 2 drops of $10 \%$ formaldehyde were added as preservative of the urine particles. Finally, $50 \mu \mathrm{l}$ of each sample was examined once a week in order to check the preservation of the particles. With this method, most samples persisted unchanged for up to 3 weeks and, therefore, were shown during the course. The samples in which lysis of particles occurred were discarded.

At the end of the course, a questionnaire, which had been prepared by one of us (F.A.), was circulated among the participants. It was aimed at assessing the professional qualification of the participants, their place of origin and their satisfaction with the course.

\section{Results}

The program carried out on day 1 was the following.

- A presentation on the methodological aspects of urine sediment preparation, examination and reporting, with emphasis on the need of using a standardized methodology (table 1). The advantages of using phase contrast microscopy versus bright field microscopy and polarized light for the identification of crystals and lipids as suggested by international guidelines was stressed $[4,5]$. This part was integrated with the projection of a video, prepared for the occasion, showing the various steps of a standardized method for the preparation of the urine samples.

- A lecture on the appearance, identification and clinical meaning of the particles of the urinary sediment,
Table 1. An example of standardized and easily reproducible methodology for urine sample preparation and examination

Simple and clear written instructions for urine collection Collection in disposable containers of the second urine of the morning after discarding the first part of micturition (= midstream urine)

Standardized centrifugation of a $10-\mathrm{ml}$ aliquot of urine at $400 \mathrm{~g}$ per $10 \mathrm{~min}$

Removal by a Pasteur pipette of $9.5 \mathrm{ml}$ of the supernatant urine Gentle but thorough resuspension of the precipitate in the remaining $0.5 \mathrm{ml}$ of urine with a Pasteur pipette

Transfer by a precision pipette of $50 \mathrm{ml}$ of the resuspended urine to a glass slide

Covering of the sample with a $24 \times 32 \mathrm{~mm}$ coverslip

Examination of the sample at the microscope on at least 20 microscopic fields

Matching of the microscopic findings with dipstick findings for $\mathrm{pH}$, specific gravity, hemoglobin, leukocyte esterase, nitrites and albumin

Report of the findings (as number of particles/milliliter or as average number/microscopic field) with a comprehensive final comment

with emphasis on the particles associated with renal diseases. This lecture also included 6 slides on urinary schistosomiasis (due to Schistosoma haematobium), which is endemic in Nigeria and whose diagnosis is based on the search of typical ova in the urine sediment.

- The presentation of 2 case reports with rapidly progressive renal disease but with quite different urine profiles, one being associated with a necrotizing glomerulonephritis and marked tubular damage, the other with scleroderma kidney.

- One practical session at the microscope during which the urine sediment of a patient with postinfectious glomerulonephritis was examined in detail.

All 3 case reports were shown with the renal biopsy findings to demonstrate the close correlations existing between the urinary findings and the renal lesions.

The program carried out on day 2 was the following.

- A presentation on the urine sediment in clinical practice, focused on isolated microscopic hematuria and on glomerular diseases.

- The presentation of 6 case reports, which offered the possibility of describing the urinary findings in a wide spectrum of diseases, namely, dysmorphic erythro- 
Table 2. Summary of the urine samples and urine particles shown during the course with their clinical associations

Nephritic sediment

Associated conditions: necrotizing GN; acute postinfectious GN; proliferative lupus nephritis

Lipiduria

Associated condition: nephrotic syndrome

Minor urine sediment changes

Associated conditions: scleroderma kidney; destructive anti-GBM disease

Dysmorphic erythrocytes including acanthocytes Associated condition: long-lasting isolated microscopic hematuria of glomerular origin

Decoy cells

Associated condition: polyoma BK virus nephropathy

Massive uric acid crystalluria

Associated condition: acute urate nephropathy due to acute leukemia

Atypical uroepithelial cells and isomorphic erythrocytes Associated conditions: bladder cancer

Birefringent spherical particles of unknown origin in a chalky urine

Associated condition: undetermined

$\mathrm{GN}=$ Glomerulonephritis; $\mathrm{GBM}=$ glomerular basement membrane.

cytes in a patient with microscopic hematuria of glomerular origin, decoy cells in a renal transplant recipient with BK virus nephropathy, massive uric acid crystalluria in a patient with acute urate nephropathy caused by acute lymphatic leukemia, minimal and unspecific urine findings in a patient with irreversible oliguric acute renal failure caused by destructive antiGBM disease, atypical uroepithelial cells and isomorphic erythrocytes in 2 patients with bladder cancer.

- One practical session at the microscope during which several particles of nephrological importance such as acanthocytes, renal tubular epithelial cells, lipids (with their typical 'Maltese cross' appearance by polarized light) and several types of cast were shown. In addition, 3 samples selected from local patients were examined: 1 with lupus nephritis, 1 with acute renal failure in a context of nephrotic syndrome and 1 with macroscopically chalky appearance of undetermined nature. This urine, which belonged to a 12 -year-old outpatient girl, was found to contain large amounts of unusual
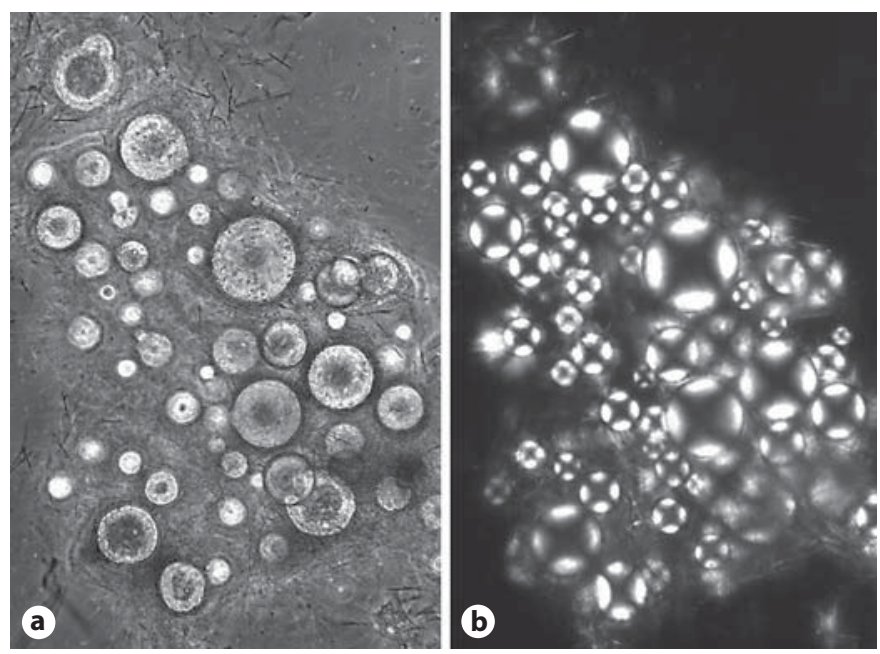

Fig. 1. a Spherical particles of different size intermingled with needle-shaped crystals (phase contrast microscopy, original magnification $\times 400$ ). b The same particles under polarized light. Their birefringence features (the arms of a 'Maltese cross' protruding from a central square) differ from 'Maltese crosses' of lipids containing free cholesterol and cholesterol esters and are of difficult interpretation (crystals? contaminants? other?).

spherical particles with peculiar birefringent features and of needle-shaped crystals, never seen before (fig. 1). This finding stimulated further investigation, which is in progress at the moment of writing 1 .

Altogether, the course encompassed the detailed description of the urine of 12 clinical cases ( 9 from Milan, 3 from Zaria) (table 2), 2 practical sessions and several discussion sessions, for a total of 10 worked hours.

The course was attended by 40 participants. By the questionnaire circulated at the end of the course, we knew that $70 \%$ of them were nephrologists or nephrology trainees (residents), while the others were, in decreasing order, pathologists, microbiologists or medical laboratory scientists. Most participants were from Northern and Western Nigeria, only 2 being from Eastern states. More than $97 \%$ of the participants found it to be very useful and practicable. All participants received a CD containing all

\footnotetext{
1 A portion of the urine was taken to Milan, where it was filtered through a Whatman 542 filter and dried. Afterwards, it was analyzed by infrared spectroscopy (Professor Michel Daudon, Hôpital Necker, Paris), which identified mainly sodium urate monohydrate, whose morphological appearances, however, are usually quite different from that observed in this case. Thus, the particles seen remain unidentified at the moment. Unfortunately, no clinical information about the patient is available.
} 
the power point presentations of the course as well as the video on urine sample preparation.

Quite unexpectedly and interestingly, during a visit to the Microbiology Department of the Ahmadu Bello Hospital at Zaria, it was found that 1 phase contrast microscope with proper magnifications was available but had never been used for urine microscopy. This finding prompted a co-operation between nephrologists and microbiologists (who also attended the course) and the setup of a urine microscopy program, which was started on 21 March with the first urine samples of renal patients being examined by nephrologists and is continuing at present [6]. Moreover, it has recently been planned that 1 nephrologist from the Zaria renal unit will spend 1 month (fixed period: October 2010) in the laboratory of the renal unit in Milan for a full training on urine microscopy.

Finally, after the course we have been informed that in other Nigerian places such as Sagamu (Ogun State), residents in nephrology are trying to start a urine sediment program in spite of 'the lack of adequate instrumentation and some hospital bureaucracy' [7].

\section{Discussion}

The Federal Republic of Nigeria is the largest nation of West Africa (surface: $923,768 \mathrm{~km}^{2}$ ) and the most populated country of the African continent (about 148 million people). The federal capital is Abuja, but the commercial nerve center is Lagos in the southwest. The North of the country is inhabited mostly by Hausa and Fulan ethnic groups, who are predominantly Muslims, while in the south Yoruba and Ibo ethnic groups predominate, who are mostly Christians [8].

Nigeria is the 6th largest oil producer in the world, however, it still belongs to the developing countries with a gross domestic product per head of 1,451 USD, a human development index of 0.499 (154th in the world ranking), an illiteracy of $28 \%$ and a life expectancy of 45 years for males and 47 years for females. Only $30 \%$ of the population have access to the health system [8].

In Nigeria, renal diseases are common. According to recent reports $[9,10]$, they represent, in children, 3.2$4.5 \%$ of all pediatric admissions, the most frequent disorders being represented by urinary tract infection, nephrotic syndrome, acute glomerulonephritis and chronic renal failure. Acute glomerulonephritis is particularly frequent in children of low social classes during the dry season and is associated with a $7.9 \%$ mortality $[11,12]$. As to chronic renal failure, a retrospective review from 1989
Table 3. The equipment required for urine microscopy

Disposable containers for urine collection (volume about $100 \mathrm{ml}$; diameter about $5.0 \mathrm{~cm}$ )

Glass graduated tubes $(10 \mathrm{ml})$ with a conical bottom

Centrifuge

Pasteur pipettes

Precision pipette

Glass slides

Glass coverslips of standardized size (e.g. $24 \times 32 \mathrm{~mm}$ )

Dipsticks for the measurement of urine $\mathrm{pH}$, specific gravity, hemoglobin, leukocyte esterase, nitrites and albumin

Microscope equipped with 2 magnification (e.g. $\times 200$ and $\times 400$ ), phase contrast device and filters for polarized light

Forms for reports

to 2003 has shown that its prevalence has greatly increased, from to 6 to $16 \%$ of all medical admissions, the 2 most frequent causes being hypertension (29.8\%) and chronic glomerulonephritis (27.8\%) [13]. Acute renal failure is also common, representing almost $20 \%$ of the admissions in an intensive care unit of Ile-Ife, in the southwest of the country, where head trauma, major burns and surgical sepsis were the most frequent conditions associated with acute kidney injury [14] - this without forgetting the cases of acute kidney injury due to nephrotoxic herbs or draughts prescribed by traditional healers [1517] or even ethylene glycol illegally used as cheap substitute solvent in drug manufacturing [18].

In spite of the high prevalence of the renal disease, the diagnostic power of Nigerian nephrologists is heavily limited by financial, technical and human resource constraints. In fact, even though renal biopsy is available in most nephrological centers, immunofluorescence and electron microscopy investigation are still unavailable, with major limitations in the management of renal patients.

Thus, the course described in this paper, which is probably the first of this kind in Sub-Saharan Africa, was organized to encourage and stimulate the practice of urine microscopy among Nigerian nephrologists and, hence, to improve their diagnostic power.

In this respect, it is worth noting that a urine microscopy program requires relatively small financial and training investments, much lower than those needed for 
other basic diagnostic techniques such as, for instance, ultrasonography or renal biopsy. In fact, urine microscopy requires very ordinary equipment and no consumable reagents (table 3 ), the only major financial investment being for the microscope, which must be of high quality and equipped with phase contrast, polarized light and 2 magnifications $(\times 200$ and $\times 400)[4,5]$. This, in Europe has a cost of about EUR 4,000 (or USD 5,000).

Thus, after the course, official representatives of the Nigerian Society of Nephrology considered that a reachable target would be that each Nigerian renal unit could set up, in a near future, its own urine microscopy program, with proper equipment (located in the renal unit itself or in cosharing with other departments) and with 1 nephrologist expert in the field, who could be trained in Milan in a 1- or 2-month period. This is because the microscopic analysis of the urine is a task of moderate complexity [19] and the microscopist of the urine should be able not only to identify all urine sediment particles, but also to put them into urinary profiles and, finally, interpret the findings in a wider clinical context.

These aspects are all very important because there is now evidence that the examination of the urine sediment by nephrologists has and added value when compared to the examination carried out by clinical pathologists working in central laboratories, far from bedside and with an inadequate knowledge of clinical correlates of urinary findings [20-22].

The results described, which demonstrate that nephrological skills can be transferred from the developed to the developing world without large financial investments, are very encouraging and are a good sign for the future.

In conclusion, we believe that training programs like the one described should be encouraged in developing countries with limited resources to further enhance their capacity to make nephrological diagnosis with the ultimate aim of improving patient care and management.

\section{Acknowledgements}

The authors thank: ISN/COMGAN for the support to the course, Mr. Emanuele Martella, Nikon Instruments SpA, Italy, for lending, free of charge, the microscope and the video camera for the course, and Mr. Andrea Centa, for the preparation of the video on methodological aspects.

\section{References}

1 Fogazzi GB, Verdesca S, Garigali G: Urinalysis: core curriculum 2008. Am J Kidney Dis 2008;51:1052-1067.

2 Fogazzi GB: The urinary sediment. An Integrated View, ed 3. Milano, Elsevier, 2009.

-3 Fogazzi GB, Garigali G, Pirovano B, Muratore MT, Raimondi S, Berti S: How to improve the teaching of urine microscopy. Clin Chem Lab Med 2007;45:407-412.

4 Kouri T, Fogazzi GB, Gant V, Hallander H, Hofmann W, Guder WG: European Urinalysis Guidelines. Scand J Clin Lab Invest 2000; 60(suppl 231):S1-S96.

5 CLSI: Document GP16-A3-Urinalysis: Approved Guideline - Third Edition (GP16A3), 2009, vol 29, No 4.

6 Bosan IB: Personal communication to Fogazzi GB.

7 Inofomoh F: Personal communication to Fogazzi GB

8 Calendario Atlante De Agostini 2010: Nigeria. Novara, Istituto Geografico De Agostini, 2009, pp 798-803.

$\checkmark 9$ Michael IO, Gabriel OE: Pattern of renal diseases in children in midwestern zone of Nigeria. Saudi J Kidney Dis Transpl 2003;14: 539-544.
10 Etuk IS, Anah MU, Ochigs SO, Eyong ME: Pattern of paediatric renal disease in inpatients in Calabar, Nigeria. Trop Doct 2006; $36: 256$.

11 Anochie I, Eke F, Okpere A: Childhood acute glomerulonephritis in Port Harcourt, Rivers State, Nigeria. Niger J Med 2009;18:162-167.

12 Etuk IS, Anah MU, Eyong ME: Epidemiology and clinical features of glomerulonephritis in Calabar, Nigeria. Niger J Physiol Sci 2009;24:91-94.

13 Arogundade FA, Barsoum RS: CKD prevention in Sub-Saharan Africa: a call for governmental, nongovernmental, and community support. Am J Kidney Dis 2008;51:515-523.

$\checkmark 14$ Okunola OO, Arogundade FA, Sanusi AA, Akinsola A: Acute renal failure in the intensive care unit: aetiological and predisposing factors and outcome. West Afr J Med 2009; 28:240-244

15 Kadiri S, Arije A, Salako BL: Traditional herbal preparations and acute renal failure in south west Nigeria. Trop Doct 1999;29:244246.

16 Adelekun TA, Ekwere TR, Akinsola A: The pattern of acute toxic nephropathy in Ife, $\mathrm{Ni}$ geria. West Afr J Med 1999;18:60-63.
17 Anochie IC, Eke FU: Acute renal failure in Nigerian children: Port Harcourt experience. Pediatr Nephrol 2005;20:1610-1614.

18 Centers for Disease Control and Prevention: Fatal poisoning among young children from diethylene glycol-contaminated acetaminophen - Nigeria, 2008-2009. Morb Mortal Wkly Rep 2009;11:1345-1347.

19 Astion AL, Kim S, Nelson A, Henderson PJ, Phillips C, Bien C, Mandel L, Orkand AR, Fine JS: A two-year study of microscopic urinalysis competency using the Urinalysis-Review Computer Program. Clin Chem 1999; 45:757-770.

20 Tsai JJ, Yeun JY, Kumar VA, Don BR: Comparison and interpretation of urinalysis performed by a nephrologist versus a hospitalbased clinical laboratory. Am J Kidney Dis 2005;46:820-829.

21 Verdesca S, Brambilla C, Garigali G, Croci MD, Messa P, Fogazzi GB: How a skilful and motivated urinary sediment examination can save the kidneys. Nephrol Dial Transplant 2007;22:1778-1781

22 Györik S, Jandus P, Marone C: Urinanalysis: a neglected but easy and inexpensive diagnostic tool. Nephrol Dial Transplant Plus 2009;2:354-356. 